\title{
Sytematic Review: The Effect of Plumbum and Zinc on Attention-Deficit/Hyperactivity Disorder (ADHD)
}

\author{
Mohammad Ladrang Pramushinto Paramanindhito ${ }^{\mathrm{a}, \mathrm{e}}$, Yunias Setiawati ${ }^{\mathrm{b}, \mathrm{f}_{*}}$, Mira \\ Irmawati $^{\mathrm{c}, \mathrm{g}}$, Citrawati Dyah Kencono Wungu ${ }^{\mathrm{d}, \mathrm{h}}$ \\ am.la.ng.p.paramanindhito-2018@fk.unair.ac.id, byunias.setiawati@ gmail.com, ${ }^{\mathrm{c}}$ irmawatimira@gmail.com, \\ d citrawati.dyah@fk.unair.ac.id
}

${ }^{\mathrm{e}}$ Faculty of Medicine, Airlangga University, Surabaya, Indonesia

${ }^{\mathrm{f}}$ Department of Medical Psychology, Faculty of Medicine, Airlangga University, Surabaya, Indonesia

${ }^{g}$ Department of Child Health, Faculty of Medicine, Airlangga University, Surabaya, Indonesia

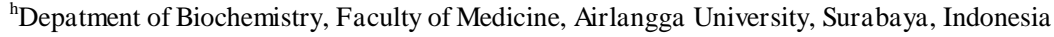

\begin{abstract}
Attention-Deficit/Hyperactivity Disorder (ADHD) is a mental disorder characterized by a lack of attention, and/or hyperactivity-impulsivity and is related to the growth and development of one's functions in carrying out social life at home and school. Attention-Deficit/Hyperactivity Disorder (ADHD) can be diagnosed in patients aged 6-18 years. The cause of ADHD is still not known for certain, but there are several factors that are considered to be the cause of ADHD, namely genetic factors, biological factors such as brain development, active neurotransmitter factors, psychosocial factors, trauma factors, family factors, emotional factors or children's temperament, and environmental factors Environmental factors can include exposure to chemicals, exposure to heavy metals, exposure to toxins, nutrition, and childbirth complications. $\mathrm{Pb}$ is a heavy metal and toxin can cause ADHD symptoms. $\mathrm{Zn}$ is a compound that when levels in the body are reduced, it can cause ADHD symptoms. $\mathrm{Pb}$ and $\mathrm{Zn}$ substances are predisposing factors for ADHD. The aim from this study is to determine the effect of $\mathrm{Pb}$ and $\mathrm{Zn}$ on Attention-Deficit/Hyperactivity Disorder. Studies was conducted through 4 e-databases, namely PubMed, ScienceDirect, Scopus, and SpringerLink. The literature quality assessment was carried out using the NOS (Newcastle-Ottawa Scale). From 1124 citations, we identified 20 relevant articles consisting of 4 cohort studies, 12 case-control studies, and 4 cross sectional studies. There are 14 studies discussing $\mathrm{Pb}$ exposure, 4 studies discussing $\mathrm{Zn}$ deficiency, and 2 studies discussing both on ADHD symptoms. Exposure to Lead $(\mathrm{Pb})$ has been shown to have an effect on ADHD symptoms especially the type of hyperactivity and impulsivity and zinc (Zn) deficiency has been shown to affect ADHD symptoms especially the inattention type.
\end{abstract}

Keywords: Lead; Plumbum; Zinc; ADHD

\section{Introduction}

Attention-Deficit/Hyperactivity Disorder (ADHD) is a disorder associated with young children characterized by inattention and/or hyperactivity-impulsivity (American Psychiatric Association, 2013; 
Barkley, 1998; Maslim, 2013). Attention-Deficit/Hyperactivity Disorder (ADHD) can be diagnosed in patients aged 6-18 years (Subcommittee on Attention-Deficit/Hyperactivity Disorder, 2011). The diagnosis of ADHD is carried out using the Diagnostic and Statistical Manual of Mental Disorder (DSM) - 5 which classifies into 3 types, namely hyperactivity - impulsivity (<15\%), inattention / neglect $(20 \%-30 \%)$, and combined (50\%-70\%) (Aprilia, 2017). The cause of ADHD is still not known for certain, but there are several factors that are considered to be the cause of ADHD, namely genetic factors, biological factors such as brain development, active neurotransmitter factors, psychosocial factors, trauma factors, family factors, emotional factors or children's temperament, and environmental factors (cigarettes, alcohol and Plumbum) (American Psychiatric Association, 2013; Permenkes, 2011; Rohmatin, 2018). Environmental factors can be in the form of exposure to chemicals, exposure to heavy metals, exposure to toxic substances, nutrition, and childbirth complications ( $\mathrm{NH}, 2017)$. Plumbum $(\mathrm{Pb})$ and Zinc (Zn) substances are predisposing factors for ADHD (Donzelli, 2019; Villagomez, 2014).

Plumbum is known as a heavy metal that is toxic. In children, the potential for toxicity symptoms due to lead exposure is higher than in adults due to high intakes per body mass, frequent insertion of foreign objects into the mouth, higher uptake, and immature body system development (Tong et al. al, 2000). Plumbum works to suppress the release of acetylcholine, dopamine, and amino acids related to calcium activity in the presynaptic section and can reduce dopamine levels by inhibiting tyrosine synthesis (Lidsky and Schneider, 2003; Setiawati, 2020). This can affect the dopamine system in the body. This substance can be found in various places such as medicine, food, water, soil, fuel, and the surrounding environment (Needleman, 1991). The reference for normal blood levels according to the Centers for Disease Control and Prevention (CDC) is $3.5 \mathrm{~g} / \mathrm{dL}$.

Zinc is known for its role in various kinds of metabolism in the body related to the performance of enzymes and proteins (Hambidge, 2000). Its role in the immune system is very important because in people with severe zinc deficiency, it can cause symptoms of recurrent infections, diarrhea, alopecia, and bullous pustular dermatitis and mental disorders (Shankar and Prasad, 1998). Decreased levels of zinc can affect the ability of cell proliferation and neuronal survival by interfering with every signaling pathway that can trigger cell apoptosis (Adamo and Oteiza, 2010). According to a reference from the Centers for Disease Control and Prevention (CDC) that normal levels of zinc in serum are $70-120 \mathrm{~g} / \mathrm{dL}$.

The levels of $\mathrm{Pb}$ and $\mathrm{Zn}$ in the body affect the function of the frontal cortex of the brain through disruption of the mechanism of action of dopamine which can cause ADHD symptoms (Barkley, 1998). Dopamine is a neurotransmitter that regulates motor skills, understanding and learning functions (Berke, 2018). Zinc plays a role in the formation of the central nervous system, the synthesis of dopamine, serotonin and norepinephrine (Setiawati, 2020). Zinc deficiency can interfere with these processes. It is suspected that low serotonin levels also can cause hyperactivity and impulsivity (Setiawati, 2020). Exposure to heavy toxic metals such as $\mathrm{Pb}$ can reduce dopamine levels by inhibiting tyrosine synthesis (Setiawati, 2020). Therefore, this study aims to examine previous research on the effect of $\mathrm{Pb}$ and $\mathrm{Zn}$ substances on ADHD.

This research is expected to provide knowledge, especially to workers who are involved with hazardous substances such as $\mathrm{Pb}$ to always use Personal Protective Equipment (PPE). The importance of giving $\mathrm{Zn}$ supplementation in children with low body and children with ADHD for clinicians. The importance of managing factory waste and other waste containing heavy metals before being disposed of to a final disposal site so as not to pollute the environment and society. 


\section{Methode}

This type of research is a qualitative systematic review. The population of this study were all published studies that discussed $\mathrm{Pb}$ and $\mathrm{Zn}$ on ADHD. The sample in this study was taken by total sampling, namely the technique of taking all samples that met the inclusion and exclusion criteria. The inclusion criteria are Contains the keywords "ADHD", "GPPH", "Lead", "Plumbum", "Pb", "Plumbum", "Zinc" and "Zn"; Research studies using English or Indonesian; Research studies describe ADHD symptoms according to the Diagnostic and Statustical Manual of Mental Disorder (DSM) - IV or DSM - 5 and the Guidelines for Classifying the Diagnosis of Mental Disorders in Indonesia III (PPDGJ III); The research design was case control, cohort and cross sectional; The age limit of subjects in the study was one to thirteen years. The exclusion criteria are The study did not use humans as research subjects; There is duplication of research studies; Research studies published over five years.

The sampling technique in this study is to collect studies available on e-databases: PubMed, ScienceDirect, Scopus, and SpringerLink. The instrument used in this research is a computer or laptop hardware device to process data. The application of the PRISMA method to avoid duplication and to process the research studies obtained to fit the inclusion criteria. The tool for assessing research studies obtained is the NOS (NewcastleOttawa Scale). After assessing the study to be reviewed, we summarize the research studies by creating a summary table containing data extraction regarding the title, author, research method, research area, research subject, and research results. At the end we analyze the result and come to a conclusion.

\section{Result}

The summary of the article screening process is presented in figure 1 . There are 20 relevant articels that consist of 4 cohort studies, 12 case-control studies, and 4 cross sectional studies. The results of the assessment of articles using NOS in each study category can be seen in tables 1, 2, and 3. The characteristics of each article can be seen in table 4.

Table 1. Newcastle-Ottawa Scale (NOS) result for cohort study

\begin{tabular}{|c|c|c|c|c|c|c|c|c|c|}
\hline \multirow[b]{2}{*}{ Study } & \multicolumn{4}{|c|}{ Selection } & \multirow[b]{2}{*}{$\begin{array}{l}\text { Comparability } \\
\text { Comparability } \\
\text { of cohorts on } \\
\text { the basis of the } \\
\text { design or } \\
\text { analysis }\end{array}$} & \multicolumn{3}{|c|}{ Outcome } & \\
\hline & $\begin{array}{l}\text { Representati } \\
\text { veness of } \\
\text { the exposed } \\
\text { cohort }\end{array}$ & $\begin{array}{l}\text { Selection } \\
\text { of the } \\
\text { non } \\
\text { exposed } \\
\text { cohort }\end{array}$ & $\begin{array}{l}\text { Ascertain } \\
\text { ment of } \\
\text { exposure }\end{array}$ & $\begin{array}{l}\text { Demonstr } \\
\text { ation that } \\
\text { outcome } \\
\text { of interest } \\
\text { was not } \\
\text { present at } \\
\text { start of } \\
\text { study }\end{array}$ & & $\begin{array}{l}\text { Assess } \\
\text { ment of } \\
\text { outcom } \\
\text { e }\end{array}$ & $\begin{array}{l}\text { Was } \\
\text { follow- } \\
\text { up } \\
\text { long } \\
\text { enough } \\
\text { for } \\
\text { outco } \\
\text { mes to } \\
\text { occur }\end{array}$ & $\begin{array}{l}\text { Adequ } \\
\text { acy of } \\
\text { follow } \\
\text { up of } \\
\text { cohorts }\end{array}$ & $\begin{array}{l}\text { Total } \\
\text { Score }\end{array}$ \\
\hline $\begin{array}{l}\text { Ji, Y., } \\
\text { et. al } \\
\text { (2018) } \\
\text { Choi, } \\
\text { W. J., } \\
\text { et. al }\end{array}$ & - & $*$ & $*$ & $*$ & $* *$ & $*$ & $*$ & $*$ & 8 \\
\hline & $*$ & $*$ & $*$ & $*$ & $* *$ & $*$ & $*$ & $*$ & 9 \\
\hline
\end{tabular}


$\mathrm{Xu}, \mathrm{J}$.

et. al

(2015)

Neuge

bauer,

J., et.

al

Table 2. Newcastle-Ottawa Scale (NOS) result for case-control study

\begin{tabular}{|c|c|c|c|c|c|c|c|c|c|}
\hline \multirow[b]{2}{*}{ Study } & \multicolumn{4}{|c|}{ Selection } & \multicolumn{2}{|l|}{ Comparability } & \multicolumn{2}{|l|}{ Outcome } & \multirow[b]{2}{*}{$\begin{array}{l}\text { Total } \\
\text { Score }\end{array}$} \\
\hline & $\begin{array}{l}\text { Is the } \\
\text { case } \\
\text { definiti } \\
\text { on } \\
\text { adequat } \\
\text { e? }\end{array}$ & $\begin{array}{l}\text { Representative } \\
\text { ness of the } \\
\text { cases }\end{array}$ & $\begin{array}{l}\text { Selecti } \\
\text { on of } \\
\text { Contro } \\
\text { ls }\end{array}$ & $\begin{array}{l}\text { Definiti } \\
\text { on of } \\
\text { Control } \\
\text { s }\end{array}$ & $\begin{array}{l}\text { Comparability } \\
\text { of cases and } \\
\text { controls on } \\
\text { the basis of } \\
\text { the design or } \\
\text { analysis }\end{array}$ & $\begin{array}{l}\text { Ascerta } \\
\text { inment } \\
\text { of } \\
\text { exposur } \\
\text { e }\end{array}$ & $\begin{array}{l}\text { Same } \\
\text { method of } \\
\text { ascertain } \\
\text { ment for } \\
\text { cases and } \\
\text { controls } \\
\end{array}$ & $\begin{array}{l}\text { Non- } \\
\text { Respo } \\
\text { nse } \\
\text { rate }\end{array}$ & \\
\hline $\begin{array}{l}\text { Elbaz, F., } \\
\text { et. al } \\
(2019) \\
\text { Park, J. }\end{array}$ & $*$ & * & - & $*$ & $* *$ & * & $*$ & * & 8 \\
\hline $\begin{array}{l}\text { H., et. al } \\
(2016) \\
\text { Lin, Y., } \\
\text { et. al }\end{array}$ & * & * & - & * & $* *$ & * & * & * & 8 \\
\hline $\begin{array}{l}\text { (2019) } \\
\text { Viktorino }\end{array}$ & * & * & - & * & * & * & $*$ & * & 7 \\
\hline $\begin{array}{l}\text { va, A., et. } \\
\text { al (2016) } \\
\text { Kim, J. }\end{array}$ & * & * & - & * & $* *$ & * & * & * & 8 \\
\hline $\begin{array}{l}\text { I., et. al } \\
\text { (2018) } \\
\text { Choi, J. }\end{array}$ & $*$ & * & - & * & $* *$ & * & * & * & 8 \\
\hline & $*$ & $*$ & - & $*$ & $*$ & $*$ & $*$ & $*$ & 7 \\
\hline $\begin{array}{l}\text { Elbaz, F., } \\
\text { et. al } \\
\text { (2017) }\end{array}$ & * & * & $*$ & $*$ & $* *$ & * & * & $*$ & 9 \\
\hline $\begin{array}{l}\text { Joo, H., } \\
\text { et. al } \\
\text { (2017) }\end{array}$ & $*$ & $*$ & * & $*$ & $*$ & $*$ & $*$ & $*$ & 8 \\
\hline $\begin{array}{l}\text { Skalny, } \\
\text { A. V., et. } \\
\text { al (2020) }\end{array}$ & $*$ & * & * & $*$ & ** & * & $*$ & * & 9 \\
\hline $\begin{array}{l}\text { Setiawati, } \\
\text { Y., et. Al }\end{array}$ & $*$ & $*$ & - & $*$ & $* *$ & * & $*$ & $*$ & 8 \\
\hline
\end{tabular}


(2019)

Hawari,

I., et. al

(2020)

Nigg, J.

T., et. al

(2016)

$*$

*

$*$

8

Table 3. Newcastle-Ottawa Scale (NOS) result for cross sectional study

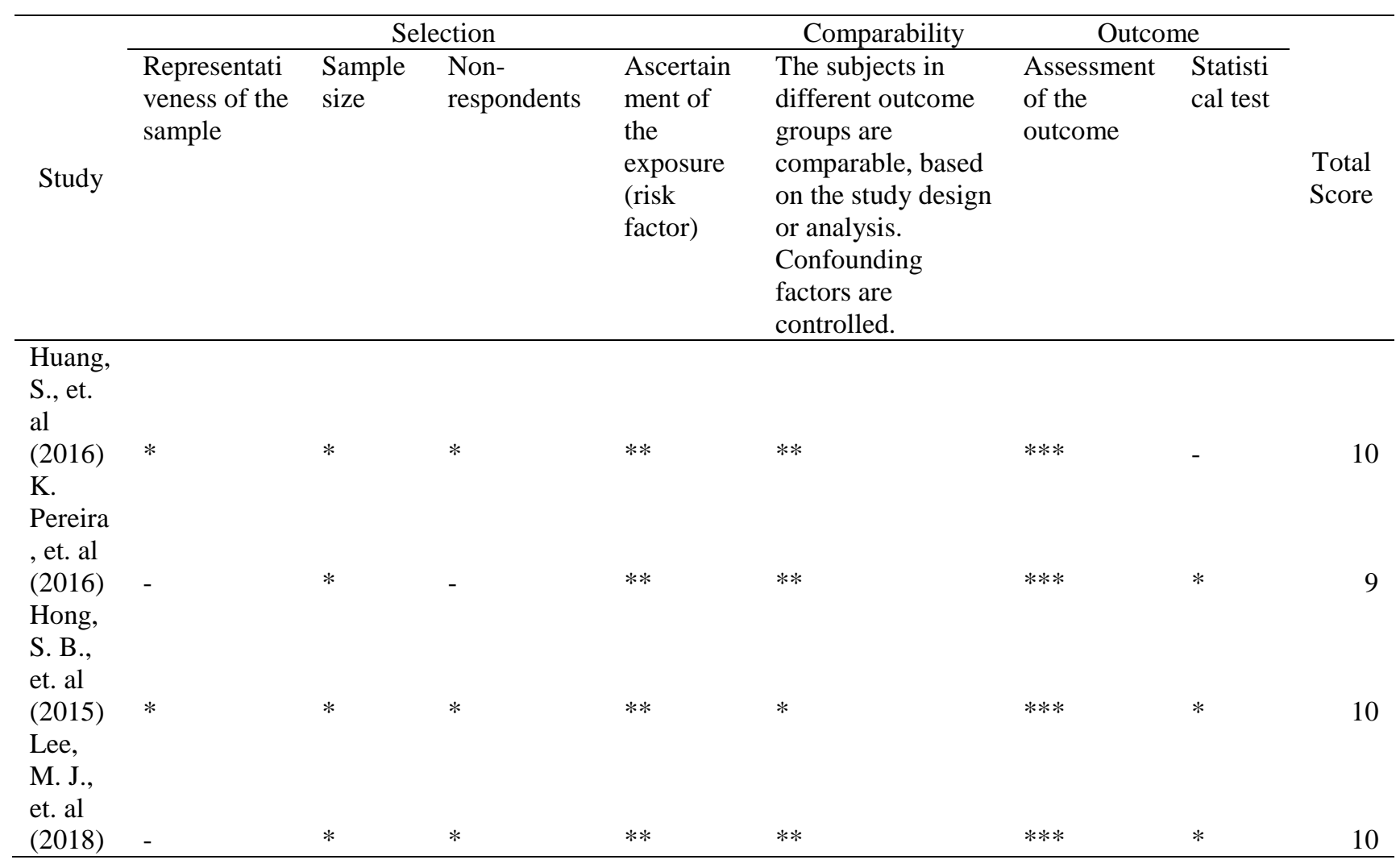

Table 4. Charateristic of the articles

\begin{tabular}{|c|c|c|c|c|c|c|c|c|}
\hline $\begin{array}{c}\text { No } \\
.\end{array}$ & $\begin{array}{c}\text { Author } \\
\text { (Year) }\end{array}$ & Title & Method & Country & Subject & $\begin{array}{l}\text { Research } \\
\text { time }\end{array}$ & Data Collection & Result \\
\hline 1 & $\begin{array}{l}\text { Ji, Y., et. } \\
\text { Al (2018) }\end{array}$ & $\begin{array}{l}\text { A Prospective } \\
\text { Birth Cohort } \\
\text { Study on Early } \\
\text { Childhood Lead } \\
\text { Levels and }\end{array}$ & $\begin{array}{l}\text { Cohort } \\
\text { study }\end{array}$ & $\begin{array}{l}\text { Boston - } \\
\text { United } \\
\text { States }\end{array}$ & $\begin{array}{l}1479 \\
\text { participan } \\
\text { ts (mean } \\
\text { age } 9.6 \\
\text { years) }\end{array}$ & 3 years & $\begin{array}{l}\text { - Non-fasting } \\
\text { blood sample } \\
\text { to measure } \\
\text { lead levels } \\
\text { - MLR }\end{array}$ & $\begin{array}{l}\text { 131 children } \\
\text { had blood } \\
\text { lead levels } \\
\text { of } 5-10 \\
\text { g/dL. }\end{array}$ \\
\hline
\end{tabular}


Attention Deficit

Hyperactivity

Disorder: New

Insight on Sex

Differences
2 Elbaz, F., Association et. al Between

(2019)

\section{Circulating}

Zinc/Ferritin

Levels and Parent

Conner's Scores

in Children with

Attention Deficit

Hyperactivity

Disorder
3 Park, J. H., Blood Lead et. al (2016)
Concentrations

and Attention

Deficit

Hyperactivity

Disorder in

Korean Children:

A Hospital-Based

Case Control

Study

$\begin{array}{lll}\text { Case- } & \text { Cairo - } & 50 \\ \text { control } & \text { Egypt } & \text { participan } \\ \text { study } & & \text { ts (43 } \\ & & \text { males, } 7 \\ & \text { females; } \\ & & \text { mean age } \\ & 9 \pm 2.2 \\ & & \text { years })\end{array}$

Case- Busan - 228

control Korea participan

study

ts $(114$

ADHD

and 114

healthy

controls;

mean age

8.7 years)
(Multivariate

Logistic

Regression)

to evaluate

the

relationship

between lead

levels and the

risk of

ADHD in

children

- Checking

blood

samples 6

months

before and

after

treatment

- Colorimetric

Test to

measure zinc

concentration

in serum

Measurement

of lead levels

in blood

samples was

carried out

using the

graphite

furnace atomic

absorption

spectrometry

method

featuring

Zeeman

background

correction
In the group

of zinc-

deficient

children

with

ADHD, data

were found

to improve

inattention

( $\mathrm{p}>0.05$ )

and

hyperactivit

y-

impulsivity

scale ( $\mathrm{p}$ >

0.001) after

taking zinc

supplementa

tion at 55

$\mathrm{mg} / \mathrm{kg}$ for 6

months.

The

geometric

mean of

blood lead

levels in the

ADHD and

control

groups were

$1.90 \pm 0.86$

$\mathrm{g} / \mathrm{dL}$ and

$1.59 \pm 0.68$

$\mathrm{g} / \mathrm{dL}$.

Measurement 


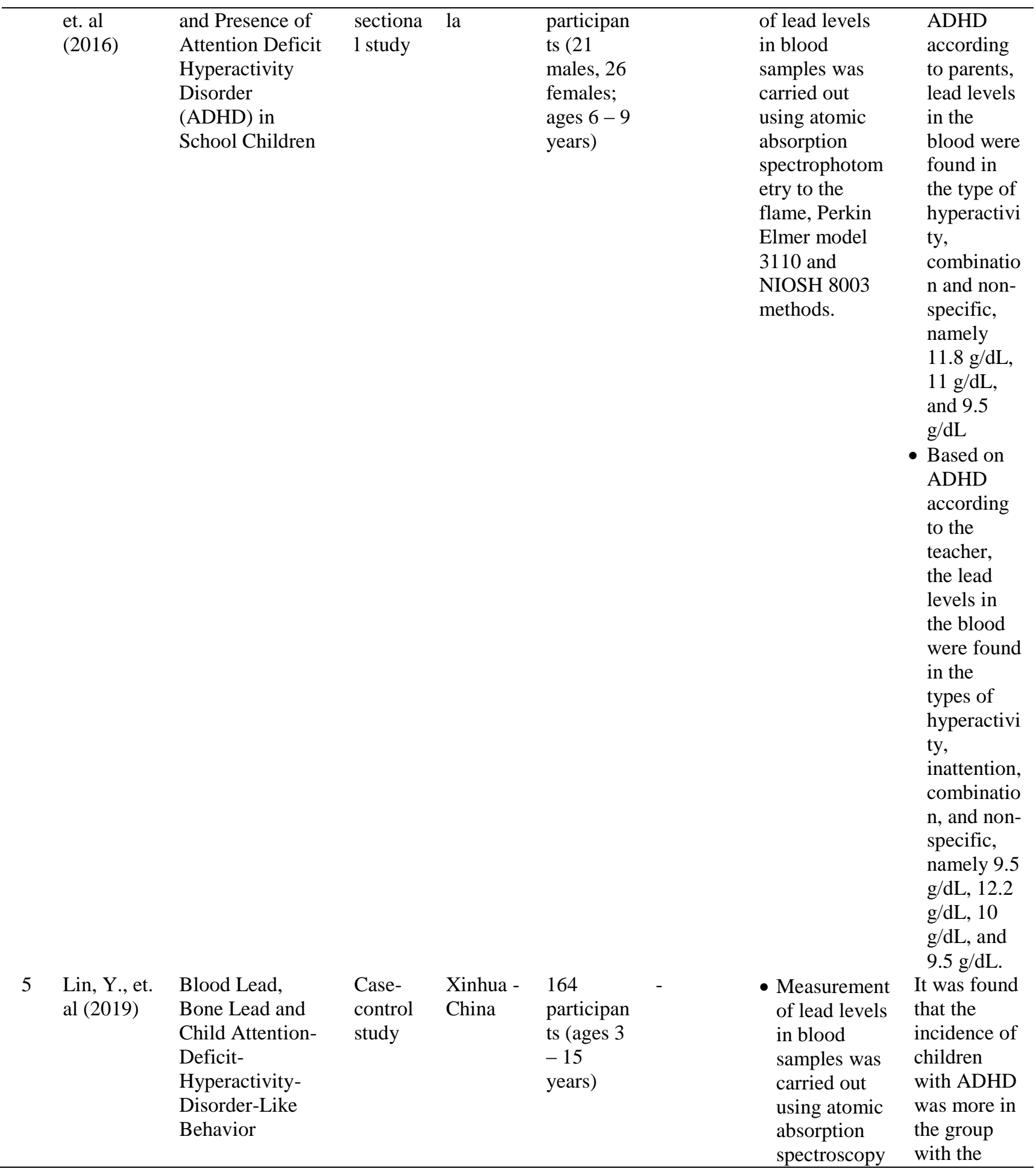




\begin{tabular}{|c|c|c|c|c|c|c|c|c|}
\hline & & & & & & & $\begin{array}{l}\text { - In vivo } \\
\text { measurement } \\
\text { of lead levels } \\
\text { in the tibia } \\
\text { bone using } \\
\text { KXFR (K- } \\
\text { shell X-ray } \\
\text { Fluorescence } \\
\text { ) }\end{array}$ & $\begin{array}{l}\text { geometric } \\
\text { mean of } \\
\text { high blood } \\
\text { and bone } \\
\text { lead levels, } \\
\text { namely } 19.6 \\
\text { g/dL and } \\
12.8 \mathrm{~g} / \mathrm{dL} .\end{array}$ \\
\hline 6 & $\begin{array}{l}\text { Choi, W. } \\
\text { J., et. al } \\
(2016)\end{array}$ & $\begin{array}{l}\text { Blood Lead, } \\
\text { Parental Marital } \\
\text { Status and The } \\
\text { Risk of Attention- } \\
\text { Deficit/Hyperacti } \\
\text { vity Disorder in } \\
\text { Elementary }\end{array}$ & $\begin{array}{l}\text { Cohort } \\
\text { study }\end{array}$ & Korea & $\begin{array}{l}2195 \\
\text { participan } \\
\text { ts (age } 7- \\
9 \text { years) }\end{array}$ & 2 years & $\begin{array}{l}\text { Measurement } \\
\text { of lead levels } \\
\text { in blood } \\
\text { samples was } \\
\text { carried out } \\
\text { using atomic } \\
\text { absorption } \\
\text { spectroscopy }\end{array}$ & $\begin{array}{l}\text { The risk of } \\
\text { ADHD } \\
\text { increases in } \\
\text { children } \\
\text { with blood } \\
\text { lead levels > } \\
2.17 \mathrm{~g} / \mathrm{dL}\end{array}$ \\
\hline 7 & $\begin{array}{l}\text { Viktorinov } \\
\text { a, A., et. al } \\
(2016)\end{array}$ & $\begin{array}{l}\text { Changed Plasma } \\
\text { Levels of Zinc } \\
\text { and Copper to } \\
\text { Zinc Ratio and } \\
\text { Their Possible } \\
\text { Associations with } \\
\text { Parent- and } \\
\text { Teacher-Rated } \\
\text { Symptoms in } \\
\text { Children with } \\
\text { Attention-Deficit } \\
\text { Hyperactivity } \\
\text { Disorder }\end{array}$ & $\begin{array}{l}\text { Case- } \\
\text { control } \\
\text { study }\end{array}$ & $\begin{array}{l}\text { Bratislav } \\
\text { a- } \\
\text { Slovakia }\end{array}$ & $\begin{array}{l}108 \\
\text { participan } \\
\text { ts (58 } \\
\text { ADHD } \\
\text { and } 50 \\
\text { healthy } \\
\text { controls; } \\
45 \text { boys } \\
\text { and } 13 \\
\text { girls; } \\
\text { mean age } \\
9.4 \pm 2.1 \\
\text { years) }\end{array}$ & - & $\begin{array}{l}\text { Measurement } \\
\text { of zinc levels } \\
\text { in plasma } \\
\text { samples was } \\
\text { carried out } \\
\text { using the flame } \\
\text { technique of } \\
\text { atomic } \\
\text { absorption } \\
\text { spectroscopy }\end{array}$ & $\begin{array}{l}\text { The mean } \\
\text { plasma zinc } \\
\text { level in the } \\
\text { ADHD } \\
\text { group was } \\
\text { lower than } \\
\text { the control } \\
\text { group (p = } \\
0.0005) \text {, } \\
\text { namely } \\
10.63 \mathrm{~g} / \mathrm{dL} \\
\text { and } 11.32 \\
\mathrm{~g} / \mathrm{dL}\end{array}$ \\
\hline 8 & $\begin{array}{l}\text { Huang, S., } \\
\text { et. al } \\
(2016)\end{array}$ & $\begin{array}{l}\text { Childhood Blood } \\
\text { Lead Levels and } \\
\text { Symptoms of } \\
\text { Attention Deficit } \\
\text { Hyperactivity } \\
\text { Disorder } \\
\text { (ADHD): A } \\
\text { Cross-Sectional } \\
\text { Study of Mexican } \\
\text { Children }\end{array}$ & $\begin{array}{l}\text { Cross } \\
\text { sectiona } \\
1 \text { study }\end{array}$ & Mexico & $\begin{array}{l}578 \\
\text { participan } \\
\text { ts (mean } \\
\text { age } 9.1 \pm \\
1.3 \text { years) }\end{array}$ & - & $\begin{array}{l}\text { Measurement } \\
\text { of lead levels } \\
\text { in blood } \\
\text { samples was } \\
\text { carried out } \\
\text { with ICP MS } \\
\text { (Inductively } \\
\text { Coupled } \\
\text { Plasma Mass } \\
\text { Spectrometry) }\end{array}$ & $\begin{array}{l}\text { The average } \\
\text { blood lead } \\
\text { level in } \\
\text { children } \\
\text { with ADHD } \\
\text { is } 3.4 \pm 2.9 \\
\mathrm{~g} / \mathrm{dL}\end{array}$ \\
\hline 9 & $\begin{array}{l}\text { Hong, S. } \\
\text { B., et. al } \\
(2015)\end{array}$ & $\begin{array}{l}\text { Environmental } \\
\text { Lead Exposure } \\
\text { and Attention } \\
\text { Defcit/Hyperactiv } \\
\text { ity Disorder } \\
\text { Symptom } \\
\text { Domains in A } \\
\text { Community }\end{array}$ & $\begin{array}{l}\text { Cross } \\
\text { sectiona } \\
1 \text { study }\end{array}$ & $\begin{array}{l}\text { South } \\
\text { Korea }\end{array}$ & $\begin{array}{l}1001 \\
\text { participan } \\
\text { ts (mean } \\
\text { age } 9.05 \\
\pm 0.7 \\
\text { years) }\end{array}$ & - & $\begin{array}{l}\text { Measurement } \\
\text { of lead levels } \\
\text { in blood } \\
\text { samples was } \\
\text { carried out } \\
\text { using an } \\
\text { atomic } \\
\text { absorption }\end{array}$ & $\begin{array}{l}\text { It was found } \\
\text { that high } \\
\text { blood lead } \\
\text { levels are } \\
\text { associated } \\
\text { with ADHD } \\
\text { in children } \\
\text { (ADHD }\end{array}$ \\
\hline
\end{tabular}


Sample of South

Korean School-

Age Children

10 Lee, M. J., Heavy Metals' et. al

Effect on

(2018)

Susceptibility to

Attention-

Deficit/Hyperacti

vity Disorder:

Implication of

Lead, Cadnium

and Antimony

11 Kim, J. I., Interaction

et. al Between DRD2

(2018)

and Lead

Exposure on The

Cortical

Thickness of The

Frontal Lobe in

Youth With

Attention-

Deficit/Hyperacti

vity Disorder

12 Choi, J.

Interaction

W., et. al

Between Lead

(2020)

and

Cross
sectiona
1 study

Taiwan

122

participan

ts (age $6-$

10 years)

$\begin{array}{lll}\text { Case- } & \text { Seoul - } & 150 \\ \text { control } & \text { South } & \text { participan } \\ \text { study } & \text { Korea } & \text { ts (75 } \\ & & \text { ADHD } \\ & & \text { and 75 } \\ & & \text { healthy } \\ & & \text { controls; } \\ & & \text { mean age } \\ & & 9.8 \text { years) }\end{array}$

Noradrenergic

Genotypes

Affects

Neurocognitive

Functions in

Attention-Deficit/

spectrometer-
graphite
furnace

Rating

Scale, 1.99;

95\% CI:

$0.17,3.81$

and 3.66;

95\% CI:

$1.18,6.13$, respectively

)

Measurement The data

of lead levels found that

in urine

samples was

carried out

using

ICP/MASS

(Inductively

Coupled

Plasma Mass

Spectrometry)

and mass

spectrometry.

Measurement

of lead levels

in blood

samples was

carried out

using an

atomic

absorption

spectrometer-

graphite

furnace

lead was

positively

associated

with ADHD

symptoms in

children ( $\mathrm{p}$

$<0.05$ )

The mean

levels of

lead in

blood

samples of

children

with ADHD

were higher

than

controls,

namely 1.5

$\mathrm{g} / \mathrm{dL}$

compared to

$1.3 \mathrm{~g} / \mathrm{dL}$

Measurement

The mean

of lead levels

in blood

samples was

carried out

using an

atomic

absorption

spectrometer-

graphite

furnace levels of

lead in

blood

samples of

children

with ADHD

were higher

than

controls,

namely 1.4

$\mathrm{g} / \mathrm{dL}$ 


\begin{tabular}{|c|c|c|c|c|c|c|c|c|}
\hline & & & & & $\begin{array}{l}\text { were } 8.8 \\
\text { and } 10.5 \\
\text { years) }\end{array}$ & & & $\begin{array}{l}\text { compared to } \\
1.3 \mathrm{~g} / \mathrm{dL}\end{array}$ \\
\hline 13 & $\begin{array}{l}\text { Elbaz, F., } \\
\text { et. al } \\
(2017)\end{array}$ & $\begin{array}{l}\text { Magnesium, Zinc } \\
\text { and Copper } \\
\text { Estimation in } \\
\text { Children with } \\
\text { Attention Deficit } \\
\text { Hyperactivity } \\
\text { Disorder } \\
\text { (ADHD) }\end{array}$ & $\begin{array}{l}\text { Case- } \\
\text { control } \\
\text { study }\end{array}$ & $\begin{array}{l}\text { Cairo - } \\
\text { Egypt }\end{array}$ & $\begin{array}{l}40 \\
\text { participan } \\
\text { ts (20 } \\
\text { ADHD } \\
\text { and } 20 \\
\text { healthy } \\
\text { controls; } \\
\text { mean age } \\
\text { of } \\
\text { children } \\
\text { with } \\
\text { ADHD } \\
\text { and } \\
\text { controls } \\
\text { were } 7.74 \\
\pm 1.48 \\
\text { and } 7.40 \\
\pm 1.35 \\
\text { years) }\end{array}$ & - & $\begin{array}{l}\text { - Measurement } \\
\text { of zinc levels } \\
\text { in serum } \\
\text { samples was } \\
\text { carried out } \\
\text { with an auto } \\
\text { analyzer } \\
\text { - Measurement } \\
\text { of zinc levels } \\
\text { in hair } \\
\text { samples was } \\
\text { carried out } \\
\text { with IC-MS } \\
\text { (Inductively } \\
\text { Coupled } \\
\text { Mass } \\
\text { Spectroscopy } \\
\text { ) and } \\
\text { compared } \\
\text { with the } \\
\text { magnesium } \\
\text { cut off value. }\end{array}$ & $\begin{array}{l}\text { - The mean } \\
\text { zinc level } \\
\text { in the } \\
\text { serum } \\
\text { sample in } \\
\text { the ADHD } \\
\text { group was } \\
\text { smaller } \\
\text { than the } \\
\text { control } \\
\text { group, } \\
\text { namely } \\
69.68 \text { g/dL } \\
\text { compared } \\
\text { to } 159.54 \\
\text { g/dL } \\
\text { The } \\
\text { number of } \\
\text { participant } \\
\text { s with zinc } \\
\text { levels } \\
\text { below the } \\
\text { cut off } \\
\text { value in } \\
\text { children } \\
\text { with } \\
\text { ADHD } \\
\text { was more } \\
\text { than } \\
\text { controls, } \\
\text { namely } 14 \\
\text { compared } \\
\text { to } 2 \\
\text { children }\end{array}$ \\
\hline 14 & $\begin{array}{l}\mathrm{Xu}, \mathrm{J} . \text {, et. } \\
\text { al (2015) }\end{array}$ & $\begin{array}{l}\text { Prenatal Lead } \\
\text { Exposure } \\
\text { Modifies the } \\
\text { Impact of } \\
\text { Maternal Self- } \\
\text { Esteem on } \\
\text { Children's } \\
\text { Inattention } \\
\text { Behavior }\end{array}$ & $\begin{array}{l}\text { Cohort } \\
\text { study }\end{array}$ & Mexico & $\begin{array}{l}192 \\
\text { participan } \\
\text { ts }(109 \\
\text { males and } \\
83 \\
\text { females; } \\
\text { mean age } \\
11.1 \pm 3.4 \\
\text { years) }\end{array}$ & 10 years & $\begin{array}{l}\text { Measurement } \\
\text { of lead levels } \\
\text { in blood } \\
\text { samples was } \\
\text { carried out } \\
\text { using } \\
\text { ICP/MASS } \\
\text { (Inductively } \\
\text { Coupled } \\
\text { Plasma Mass } \\
\text { Spectrometry) }\end{array}$ & $\begin{array}{l}\text { The } \\
\text { geometric } \\
\text { mean of } \\
\text { blood lead } \\
\text { levels in } \\
\text { children } \\
\text { with ADHD } \\
\text { is } 2.8 \mathrm{~g} / \mathrm{dL}\end{array}$ \\
\hline
\end{tabular}




\begin{tabular}{|c|c|c|c|c|c|c|c|c|}
\hline 15 & $\begin{array}{l}\text { Joo, H., et. } \\
\text { al (2017) }\end{array}$ & $\begin{array}{l}\text { Secondhand } \\
\text { Smoke Exposure } \\
\text { and Low Blood } \\
\text { Lead Levels in } \\
\text { Association With } \\
\text { Attention-Deficit } \\
\text { Hyperactivity } \\
\text { Disorder and Its } \\
\text { Symptom } \\
\text { Domain in } \\
\text { Children: A } \\
\text { Community- } \\
\text { Based Case- } \\
\text { Control Study }\end{array}$ & $\begin{array}{l}\text { Case- } \\
\text { control } \\
\text { study }\end{array}$ & $\begin{array}{l}\text { Cheonan } \\
\text { - South } \\
\text { Korea }\end{array}$ & $\begin{array}{l}428 \\
\text { participan } \\
\text { ts }(214 \\
\text { ADHD } \\
\text { and } 214 \\
\text { healthy } \\
\text { controls; } \\
\text { ages } 6- \\
10 \text { years) }\end{array}$ & - & $\begin{array}{l}\text { Measurement } \\
\text { of lead levels } \\
\text { in blood } \\
\text { samples was } \\
\text { carried out by } \\
\text { atomic } \\
\text { absorption } \\
\text { spectrophotom } \\
\text { etry }\end{array}$ & $\begin{array}{l}\text { The mean } \\
\text { blood lead } \\
\text { levels in } \\
\text { children } \\
\text { with ADHD } \\
\text { were higher } \\
\text { than } \\
\text { controls, } \\
\text { namely } 1.65 \\
\text { g/dL } \\
\text { compared to } \\
1.49 \mathrm{~g} / \mathrm{dL}\end{array}$ \\
\hline 16 & $\begin{array}{l}\text { Skalny, A. } \\
\text { V., et. al } \\
(2020)\end{array}$ & $\begin{array}{l}\text { Serum Zinc, } \\
\text { Copper, Zinc-to- } \\
\text { Copper Ratio, } \\
\text { and Other } \\
\text { Essential } \\
\text { Elements And } \\
\text { Minerals in } \\
\text { Children with } \\
\text { Attention } \\
\text { Deficit/Hyperacti } \\
\text { vity Disorder } \\
\text { (ADHD) }\end{array}$ & $\begin{array}{l}\text { Case- } \\
\text { control } \\
\text { study }\end{array}$ & Russia & $\begin{array}{l}136 \\
\text { participan } \\
\text { ts (68 } \\
\text { ADHD } \\
\text { and } 68 \\
\text { neurotypi } \\
\text { cal } \\
\text { controls; } \\
\text { ages } 4-9 \\
\text { years) }\end{array}$ & - & $\begin{array}{l}\text { Measurement } \\
\text { of zinc levels } \\
\text { in serum } \\
\text { samples was } \\
\text { carried out by } \\
\text { inductively- } \\
\text { coupled plasma } \\
\text { mass } \\
\text { spectrometry at } \\
\text { NexION 300D } \\
\text { plus an ESI } \\
\text { SC-2 DX4 } \\
\text { autosampler. }\end{array}$ & $\begin{array}{l}\text { The mean } \\
\text { serum zinc } \\
\text { level in } \\
\text { children } \\
\text { with ADHD } \\
\text { was lower } \\
\text { than in } \\
\text { neurotypical } \\
\text { controls at } \\
0.93 \pm 0.101 \\
\text { g/mL } \\
\text { compared to } \\
1.007 \pm \\
0.166 \mathrm{~g} / \mathrm{mL}\end{array}$ \\
\hline 17 & $\begin{array}{l}\text { Setiawati, } \\
\text { Y., et. Al } \\
(2019)\end{array}$ & $\begin{array}{l}\text { The Influence Of } \\
\text { Lead }(\mathrm{Pb}) \text {, Zinc } \\
(\mathrm{Zn}) \text {, Ratio Lead } \\
(\mathrm{Pb}) \text { to Zinc }(\mathrm{Zn}) \\
\text { in Attention } \\
\text { Deficit } \\
\text { Hyperactivity } \\
\text { Disorder } \\
\text { (ADHD) }\end{array}$ & $\begin{array}{l}\text { Case- } \\
\text { control } \\
\text { study }\end{array}$ & $\begin{array}{l}\text { Surabay } \\
\text { a - } \\
\text { Indonesi } \\
\text { a }\end{array}$ & $\begin{array}{l}44 \\
\text { participan } \\
\text { ts }(23 \\
\text { ADHD } \\
\text { and } 21 \\
\text { non- } \\
\text { ADHD; } \\
\text { ages } 6-9 \\
\text { years) }\end{array}$ & - & $\begin{array}{l}\text { Measurement } \\
\text { of lead and } \\
\text { zinc levels in } \\
\text { hair samples } \\
\text { was carried out } \\
\text { by atomic } \\
\text { absorption } \\
\text { spectrophotom } \\
\text { etry }\end{array}$ & $\begin{array}{l}\text { The results } \\
\text { showed } \\
\text { that there } \\
\text { was no } \\
\text { significant } \\
\text { difference } \\
\text { in lead } \\
\text { levels in } \\
\text { hair } \\
\text { samples } \\
\text { between } \\
\text { the ADHD } \\
\text { and non- } \\
\text { ADHD } \\
\text { groups } \\
\text { with } \\
\text { median } \\
\text { values of } \\
10.74 \text { and } \\
11.39 \text {. }\end{array}$ \\
\hline
\end{tabular}


18 Neugebau The Influence of er, J., et. al (2015)

\section{Low Level Pre- and Perinatal} Exposure To PCDD/Fs, Pcbs, and Lead on Attention Performance and Attention-Related Behavior Among German SchoolAged Children: Results From The Duisburg Birth Cohort Study

19 Hawari, I., The Role of Lead, et. al (2020)
Manganese, and Zinc in Autism Spectrum Disorders (ASDs) and Attention-

Deficient Hyperactivity Disorder (ADHD): a CaseControl Study on Syrian Children Affected by the Syrian Crisis

$\begin{array}{lll}\text { Cohort } & \text { Duisbur } & 117 \\ \text { study } & \mathrm{g}- & \text { participan } \\ & \text { German } & \text { ts (63 } \\ & \mathrm{y} & \text { males and } \\ & & 54 \\ & & \text { females; } \\ & & \text { mean age } \\ & & 8.5 \pm 0.3 \\ & & \text { years })\end{array}$

$\begin{array}{lll}\begin{array}{l}\text { Case- } \\ \text { control }\end{array} & \text { Syria } & 101 \\ \text { study } & \text { participan } \\ & \text { ts (31 } \\ & \text { ASD, 29 } \\ & \text { ADHD, } \\ & 11 \text { ADHD } \\ & \text { comorbid } \\ & \text { ASD, and } \\ & 30 \\ & \text { healthy } \\ & \text { controls; } \\ & \text { ages 3- } \\ & 12 \text { years })\end{array}$

- The median of zinc levels in children with ADHD and nonADHD

had a significant difference, namely 307.84 and 177.55

Measurement of the level of lead in blood samples in prenatal mothers was carried out by atomic absorption spectroscopy

The results obtained mean levels of lead in maternal blood samples during prenatal period of $22.16 \mathrm{~g} / \mathrm{L}$

- Measurement of lead levels in blood samples was carried out with an atomic absorption spectrometer

- Measurement of zinc levels in serum samples was carried out with an Olympus - The mean levels of lead in blood samples in children with ADHD were higher than controls, namely $3.243 \pm$ $0.855 \mathrm{~g} / \mathrm{dL}$ compared

to $2.84 \pm$ AU400 auto $\quad 0.7 \mathrm{~g} / \mathrm{dL}$ analyzer - The mean coupled with 
20 Nigg, J. Variation in an T., et. al Iron Metabolism (2016) Gene Moderates the Association Between Blood Lead Levels and Attention-

Deficit/Hyperacti vity Disorder in Children

$\begin{array}{lll}\text { Case- } & \text { Michiga } & 269 \\ \text { control } & \mathrm{n}- & \text { participan } \\ \text { study } & \text { America } & \text { ts (122 } \\ & & \text { ADHD } \\ & \text { and 147 } \\ & \text { non- } \\ & \text { ADHD; } \\ & & \text { mean age } \\ & \text { group for } \\ & \text { ADHD } \\ & \text { and non- } \\ & \text { ADHD } \\ & \text { were 12.5 } \\ & \text { and 11.5 } \\ & & \text { years) }\end{array}$

Measurement of lead levels in blood samples was carried out using inductively coupled plasma mass in serum wavelength samples in diffraction grating spectrophoto metry. children with ADHD was higher than controls, namely $83.45 \pm$ $13.42 \mathrm{~g} / \mathrm{dL}$ compared to $79.97 \pm$ $13.72 \mathrm{~g} / \mathrm{dL}$ The mean levels of lead in blood samples in children with ADHD were higher than nonspectrometry ADHD, namely 0.94 $\mathrm{g} / \mathrm{dL}$ compared to $0.74 \mathrm{~g} / \mathrm{dL}$ 


\section{Identification of studies via databases and registers}

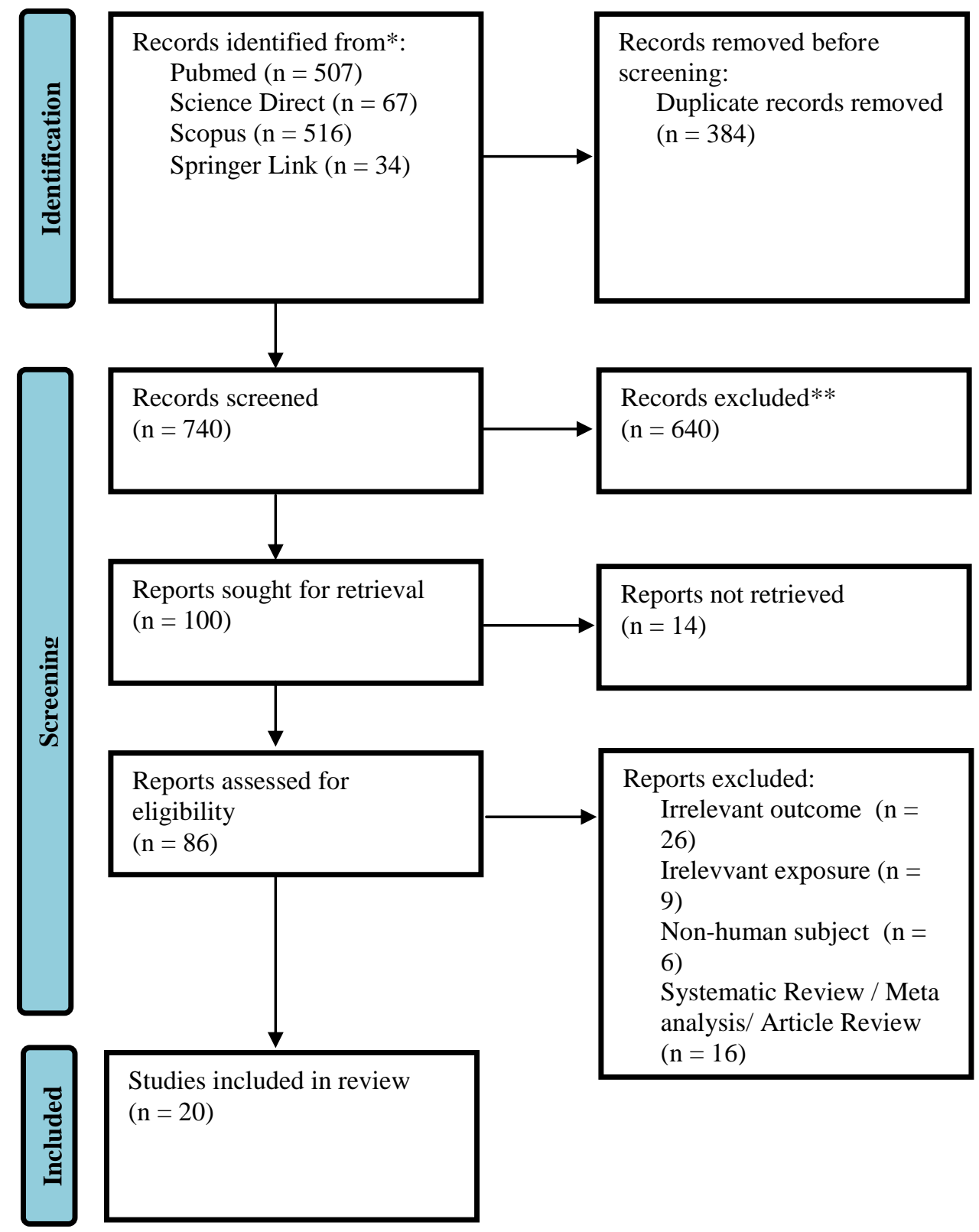

Figure 1. PRISMA flow chart articles processing 


\section{Discussion}

The results of the studies involved in this study showed evidence that Plumbum exposure was positively associated with the risk of developing ADHD, especially the types of hyperactivity and impulsivity. There is no reference to the minimum level of lead in the blood that can cause ADHD symptoms in children. The zinc category provides evidence that people with ADHD have lower zinc levels in the studies involved. As is well known, Attention-Deficit/Hyperactivity Disorder (ADHD) or Attention Deficit Disorder and Hyperactivity $(\mathrm{GPPH})$ is a persistent neurodevelopmental disorder with a prevalence of $5 \%$ in children and $2.5 \%$ in adults in worldwide (Faraone, et. al, 2015). This systematic review expands knowledge about the influence of $\mathrm{Pb}$ and Zn on ADHD because it includes the most recent studies published in 2015-2020. The results of this study support the previous review regarding Plumbum exposure to ADHD which was limited to articles with the year published until 2018. There are very few systematic review articles on Zinc, so this research can add insight on the role of Zinc on ADHD symptoms.

Of the 20 studies discussed, there are 15 studies that support the theory of $\mathrm{Pb}$ exposure to ADHD symptoms and 5 studies that support the theory of lack of Zn levels on ADHD symptoms. These studies consisted of 4 cohort studies, 12 case-control studies, and 4 cross-sectional studies which broadens the scope of the topic of this research. In this study, it was shown that $\mathrm{Pb}$ levels in ADHD sufferers increased and exposure to small amounts could lead to an increased risk of ADHD. In the Zn category, there is evidence that levels are low in ADHD sufferers and are correlated with inattention subtypes that will improve with supplementation. The studies that have been reviewed are strong enough because the studies presented are published studies from good, official literature, and have been peer reviewed before publication.

Plumbum $(\mathrm{Pb})$ has been widely known to be a neurotoxic substance for the nervous system with mechanisms related to the release of neurotransmitters and dopamine at the presynaptic (Bressler and Goldstein, 1991; Lidsky and Schneider, 2003). The Centers for Disease Control and Prevention (CDC) states that normal levels of lead in the blood are around $3.5 \mathrm{~g} / \mathrm{dL}$. Based on this systematic review, there were no significant results that levels that exceeded the new normal reference would cause ADHD symptoms in children. Other results show that there is a positive relationship between lead exposure from the environment with low levels that can affect levels in the body and increase ADHD symptoms in children. Exposure to lead which is neurotoxic from the environment can be mixed in the form of air, food and water (Dórea, J.G., 2019). Several studies in this study did not include whether the subjects who were part of the study received lead exposure in a certain period of time or in a certain form of exposure.

The role of Zinc $(\mathrm{Zn})$ in the human body has an important role in protein synthesis, DNA synthesis, the body's immune system, growth - development, synaptic transmission, signaling cascades, and regulates the production of neuronal oxidant cells in the nervous system (Adamo and Oteiza, 2010; Villagomez and Ramtekkar, 2014). Zinc plays an important role in the production and regulation of melatonin whose job it is to modulate the dopamine system, which is impaired in children with ADHD (Villagomez and Ramtekkar, 2014). In addition, zinc can help regulate and bind to the dopamine transporter for distribution. In this systematic review, the results were not significant. All studies in this study were case-control studies, most of which showed that serum zinc levels in children with ADHD were lower than normal controls. The Centers for Disease Control and Prevention (CDC) states that the normal level of zinc in serum is 70-120 g/dL. One study showed that zinc levels in children with ADHD exceeded the normal reference set by the CDC. It is proven in one study that zinc supplementation can improve ADHD symptoms in children, especially the 
inattentive type. This systematic review supports previously published research that decreased or increased zinc levels can influence ADHD symptoms (Colquhoun and Bunday, 1981).

The quality and evidence presented by the studies is strong enough and is based on the Diagnostic and Statistical Manual of Mental Disorders edition 4 or 5 , but because the country of origin of each study is different and the respondents are different, the ADHD diagnostic tools used are different in each study. Several studies have not considered separating the subtypes of ADHD symptoms into inattention and hyperactivity-impulsivity. The limitation of the language used in this study, namely English, causes the possibility of not covering research that is relevant to other languages. The existence of confounding variables that differ in each study such as age, gender, parental marital status, maternal stress level, maternal lipoprotein levels, and others cause possible bias in this review.

\section{Conclusion and Sugesstions}

Of all the studies that have been reviewed in this study, Plumbum $(\mathrm{Pb})$ exposure has been shown to have an effect on ADHD symptoms, especially the type of hyperactivity and impulsivity, although in small levels and zinc $(\mathrm{Zn})$ deficiency has been shown to affect ADHD symptoms, especially the inattention type. Future research is expected to consider the separation of ADHD symptoms in each of its subtypes. It is hoped that in the future further research will be carried out regarding the minimum reference for lead and zinc levels in relation to increasing ADHD symptoms in children and more research that will reveal the effects of $\mathrm{Pb}$ exposure and Zn deficiency on ADHD symptoms, especially in Indonesia.

\section{References}

Adamo, A. M., \& Oteiza, P. I. (2010). Zinc deficiency and neurodevelopment: the case of neurons. Biofactors, 36(2), 117-124.

Aguiar, A., Eubig, P. A., \& Schantz, S. L. (2010). Attention Deficit/Hyperactivity Disorder: A Focused Overview For Children's Environmental Health Researchers. Environmental health perspectives, 118(12), 1646-1653.

American Psychiatric Association. (2013). Diagnostic and statistical manual of mental disorders (DSM-5®). American Psychiatric Pub.

Aprilia, E., \& Oktaria, D. (2017). Kemampuan Akademik Penderita Attention Deficit-Hyperactivity Disorder (ADHD) pada Tingkat Perguruan Tinggi. Jurnal Majority, 7(1), 164-168.

Barkley, R. A. (1998). Attention-Deficit Hyperactivity Disorder. Scientific American, 279(3), 66-71.

Berke, J. D. (2018). What Does Dopamine Mean?. Nature neuroscience, 21(6), 787-793.

Bressler, J. P., \& Goldstein, G. W. (1991). Mechanisms of lead neurotoxicity. Biochemical pharmacology, 41(4), 479-484.

CDC. (2004). Preventing Lead Exposure in Young Children: A Housing-Based Approach to Primary Prevention of Lead Poisoning. https://www.cdc.gov/nceh/lead/publications/primary\%20prevention\%20document.pdf

CDC. (2019). Attention-Deficit/Hyperactivity Disorder (ADHD): Data and Statistics About ADHD. https://www.cdc.gov/ncbddd/ADHD/data.html

CDC. (2021). Childhood Lead Poisoning Prevention: Blood Lead Level in Children. https://www.cdc.gov/nceh/lead/prevention/blood-lead-levels.ht

Colquhoun, I., \& Bunday, S. (1981). A lack of essential fatty acids as a possible cause of hyperactivity in children. Medical hypotheses, 7(5), 673-679.

Daneshparvar, M., Mostafavi, S. A., Jeddi, M. Z., Yunesian, M., Mesdaghinia, A., Mahvi, A. H., \& 
Akhondzadeh, S. (2016). The Role Of Lead Exposure On Attention- Deficit/Hyperactivity Disorder In Children: A Systematic Review. Iranian journal of psychiatry, 11(1), 1.

Donzelli, G., Carducci, A., Llopis-Gonzalez, A., Verani, M., Llopis-Morales, A., Cioni, L., \& MoralesSuárez-Varela, M. (2019). The Association Between Lead And Attention-Deficit/Hyperactivity Disorder: A Systematic Review. International journal of environmental research and public health, 16(3), 382.

Dórea, J. G. (2019). Environmental exposure to low-level lead $(\mathrm{Pb})$ co-occurring with other neurotoxicants in early life and neurodevelopment of children. Environmental research, 177, 108641.

Eubig, P. A., Aguiar, A., \& Schantz, S. L. (2010). Lead and PCBs as Risk Factors for Attention Deficit/Hyperactivity Disorder. Environmental health perspectives, 118(12), 1654-1667.

Faraone, S. V., Asherson, P., Banaschewski, T., Biederman, J., Buitelaar, J. K., Ramos-Quiroga, J. A., Rohde, L. A., Sonuga-Barke, E. J., Tannock, R., \& Franke, B. (2015). Attention-deficit/hyperactivity disorder, Nature reviews, Disease primers, 1, 15020. https://doi.org/10.1038/nrdp.2015.20

Hambidge, M. (2000). Human zinc deficiency. The Journal of nutrition, 130(5), 1344S-1349S.

Hariri, M., \& Azadbakht, L. (2015). Magnesium, Iron, and Zinc Supplementation for The Treatment Of Attention Deficit Hyperactivity Disorder: A Systematic Review On The Recent Literature. International journal of preventive medicine, 6 .

Janka, Z. (2019). Tracing trace elements in mental functions, Ideggyogyaszati szemle, 72(11-12), 367-379.

Jaisoorya, T. S., Desai, G., Nair, B., Rani, A., Menon, P. G., \& Thennarasu, K. (2019). Association Of Childhood Attention Deficit Hyperactivity Disorder Symptoms With Academic And Psychopathological Outcomes In Indian College Students: A Retrospective Survey. East Asian Archives of Psychiatry, 29(4), 124.

Lidsky, T. I., \& Schneider, J. S. (2003). Lead neurotoxicity in children: basic mechanisms and clinical correlates, Brain, 126(1), 5-19.

Marshall, R., Neill, P., \& Theodosiou, L. (2011). Prevalence Of Attention Deficit Hyperactivity Symptoms In Parents Of Children Diagnosed With The Condition. Procedia-Social and Behavioral Sciences, 15, 30563058.

Maslim, R. (2013). Diagnosis Gangguan Jiwa Rujukan Ringkas dari PPDGJ-III dan DSM-5. Jakarta: PT Nuh Jaya.

Needleman, H. L. (1991). Human lead exposure. CRC Press.

NH., F. A., and Setiawati, Y. (2017). Interaksi Faktor Genetik dan Lingkungan pada Attention Deficit/Hyperactivity Disorder (ADHD). Jurnal Psikiatri Surabaya, 6(2), 42-51.

Nurmadilla, N. (2015). Potensi Zinc dalam Tatalaksana Berbagai Penyakit. https://www.researchgate.net/publication/326833922_Potensi_Zinc_dalam_Tatalaksana_Berbagai_Penyak it/link/5ba44fc892851ca9ed1a1531/download

Nuryanti, L. (2008). Psikologi Anak. Jakarta: PT. Indeks.

Peraturan Menteri Kesehatan Republik Indonesia Nomor 330/Menkes/Per/II/2011. Pedoman Deteksi Dini Gangguan Pemusatan Perhatian dan Hiperaktivitas (GPPH) pada Anak serta Penanganannya. 17 Februari 2011. Berita Negara Republik Indonesia Tahun 2011 Nomor 107. Jakarta.

Pineda-Cirera, L., Shivalikanjli, A., Cabana-Domínguez, J., Demontis, D., Rajagopal, V. M., Børglum, A. D., Faraone, S. V., Cormand, B., \& Fernàndez-Castillo, N. (2019). Exploring Genetic Variation That Influences Brain Methylation in Attention-Deficit/Hyperactivity Disorder. Translational psychiatry, 9(1), $1-11$.

Rohmatin, T. (2018). Perkembangan Potensi Akademik Anak ADHD Di Sekolah. uiltuk lnah Inhlusi.

Salehi, B., Mohammadbeigi, A., Sheykholeslam, H., Moshiri, E., \& Dorreh, F. (2016) Omega-3 and Zinc Supplementation As Complementary Therapies In Children With Attention-Deficit/Hyperactivity Disorder. Journal of research in pharmacy practice, 5(1), 22.

Setiawati, Y., Mukono, H. J., Wahyuhadi, J., \& Warsiki, E. (2019). The Influence of Lead (Pb), Zinc (Zn), Ratio Lead $(\mathrm{Pb})$ to Zinc $(\mathrm{Zn})$ in Attention Deficit Hyperactivity Disorder (ADHD). Indian Journal of 
Public Health Research \& Development, 10(8), 1497-1502.

Setiawati, Y., Mukono, H. J., Wahyuhadi, J., Warsiki, E., \& Yuniar, S. (2020). Is there an Effect of Serotonin on Attention Deficit Hyperactivity Disorder. EXECUTIVE EDITOR, 11(01), 1745.

Setiawati, Y., Wahyuhadi, J., Mukono, H. J., Warsiki, E., Fitriyah, I., Dwiyatna, A. A., \& Dharmawan, A. (2020) The Effect of Plumbum, Zinc and Zinc Ratio on Plumbum in Children's Temperament. Medico Legal Update, 20(2), 801-806.

Shankar, A. H., \& Prasad, A. S. (1998). Zinc and immune function: the biological basis of altered resistance to infection. The American journal of clinical nutrition, 68(2), 447S-463S.

Subcommittee on Attention-Deficit/Hyperactivity Disorder, Steering Committee on Quality Improvement and Management, Wolraich, M., Brown, L., Brown, R. T., DuPaul, G., Earls, M., Feldman, H. M., Ganiats, T. G., Kaplanek, B., Meyer, B., Perrin, J., Pierce, K., Reiff, M., Stein, M. T., \& Visser, S. (2011). ADHD: Clinical Practice Guideline For The Diagnosis, Evaluation, And Treatment Of AttentionDeficit/Hyperactivity Disorder In Children And Adolescents. Pediatrics, 128(5), 1007-1022.

Sudirman, A. (2017). Hubungan Pola Konsumsi Zinc (Zn) Dengan Kadar Zinc (Zn) Serum Dan Outcome Kehamilan Pada Ibu Post Partum Di Rumah Sakit Ibu Dan Anak. http://digilib.unhas.ac.id/uploaded_files/temporary/DigitalCollection/YmQxNzAwMmQ2ZGIzYWMxY2J jOWI1OTRiZTImNGNINTFhZTZhNTc0Ng==.pdf

Suprihatin, T. (2018). Modifikasi Perilaku Untuk Meningkatkan Perilaku Memperhatikan Pada Siswa Sd Yang Mengalami Gejala Gangguan Pemusatan Perhatian Dan Hiperaktivitas (GPPH). Proyeksi: Jurnal Psikologi, 9(2), 15-36.

Thomas, R., Sanders, S., Doust, J., Beller, E., \& Glasziou, P. (2015) Prevalence of AttentionDeficit/Hyperactivity Disorder: A Systematic Review and Meta-Analysis. Pediatrics, 135(4), e994-e1001.

Tong, S., Schirnding, Y. E. V. \& Prapamontol, T. (2000). Environmental lead exposure: a public health problem of global dimensions. Bulletin of the world health organization, 78, 1068-1077.

Villagomez, A., \& Ramtekkar, U. (2014). Iron, Magnesium, Vitamin D, and Zinc Deficiencies in Children Presenting With Symptoms of Attention-Deficit/Hyperactivity Disorder. Children, 1(3), 261-279.

Widhyari, S. D. (2012). Peran Dan Dampak Defisiensi Zinc (Zn) Terhadap Sistem Tanggap Kebal. Wartazoa, 22(3), 141-148. 